\title{
Participação em aulas de Educação Física e atitudes positivas para a prática de atividade física em adolescentes do estado de Sergipe, Brasil
}

Participation in Physical Education classes and positive attitudes towards physical activity in adolescents in the Brazilian State of Sergipe, Brazil

\author{
F. M. de Almeida Silva ${ }^{1,3^{*}}$; A. Smith-Menezes ${ }^{1,2,3}$ \\ ${ }^{1}$ Universidade Federal de Sergipe, Programa de Pós - Graduação em Educação Física - PPGEF. Aracaju, Sergipe. \\ ${ }^{2}$ Instituto Federal de Educação, Ciência e Tecnologia de Sergipe. \\ ${ }^{3}$ Grupo de Pesquisa em Educação Física e Saúde (GPEFiS), Aracaju, Sergipe.
}

*fabianasilvaedf@gmail.com

(Recebido em 11 de fevereiro de 2016; aceito em 27 de junho de 2016)

\begin{abstract}
A participação nas aulas de Educação Física pode oferecer oportunidades aos adolescentes de experimentar e de apreciar um estilo de vida fisicamente ativo. Analisar a associação entre participação em aulas de Educação Física e atitudes positivas para a prática de atividade física em adolescentes. Trata-se de um estudo transversal de base escolar com amostra representativa de estudantes do ensino médio da rede pública estadual de Sergipe ( $\mathrm{n}=3.992,14$ a 19 anos de idade). Os dados foram coletados mediante questionário auto administrado. Foram analisadas atitudes como: realizar atividade física no tempo livre e preferir atividades de lazer fisicamente ativas a atividades sedentárias. Recorreu-se à regressão logística binária para análise de associação entre as variáveis. Os adolescentes que relataram participar das aulas de Educação Física apresentaram mais chances de preferir atividades de lazer fisicamente ativas $(\mathrm{OR}=1,69$; $1,45-1,96)$ e realizar atividades físicas no tempo livre $(\mathrm{OR}=2,00 ; 1,72-2,31)$. Os resultados indicam que a participação nas aulas de Educação Física pode ser um fator importante para reduzir a inatividade física entre os adolescentes.
\end{abstract}

Palavras-chave: Atividade Motora, Educação Física, Adolescentes

Participation in Physical Education classes can provide opportunities for adolescents to experience and enjoy a physically active lifestyle. To analyze the association between participation in Physical Education classes and positive attitudes towards physical activity in adolescents. This is a cross-sectional school-based study with sample representative of high-school students of the public network of Sergipe ( $n=3.992,14$ 19 years old). Data were collected through a self-administered questionnaire. The following attitudes were analyzed: performance of physical activity during leisure time and preference for physically active leisure activities rather than sedentary activities. Binary logistic regression was used to analyze association among variables. Adolescents who reported participating in Physical Education classes were more likely to prefer physically active leisure activities $(\mathrm{OR}=1,69 ; 1,45-1,96)$ and engage in physical activity during leisure time $(\mathrm{OR}=2,00 ; 1,72-2,31)$. The results indicate that participation in Physical Education classes can be an important factor in reducing physical inactivity among adolescents.

Keywords: Physical Activity, Physical Education, Teenager

\section{INTRODUÇÃO}

A prática regular de atividade física durante a adolescência está associada à saúde esquelética (conteúdo mineral e densidade óssea) [1] e ao controle da obesidade [2]. Além disso, existe uma relação inversa entre o nível de atividade física e o risco de ocorrência de diversas doenças crônicas não transmissíveis, como o câncer e a hipertensão [3], que embora os seus efeitos nocivos manifestem na idade adulta está cada vez mais compreendido que o desenvolvimento começa na infância e adolescência [4].

Estudos demonstram que a adoção de um estilo de vida ativo pode ser mais facilmente incorporada na idade jovem, e que a participação em programas de exercícios na idade adulta está associada a comportamentos adquiridos em idades precoces [1,5]. No entanto, a disponibilidade 
de tecnologia, o aumento da insegurança e diminuição dos espaços livres para a prática de atividade física entre outros fatores motivacionais e de apoio familiar têm contribuído para um estilo de vida menos ativo entre os jovens [6].

Entretanto, evidências consistentes têm revelado que a participação em aulas de Educação Física está diretamente associada ao engajamento em atividades físicas fora do ambiente escolar [7,8], assim como redução da exposição ao comportamento sedentário [7]. Além disso, a participação em aulas de Educação Física pode contribuir para o alcance dos níveis recomendados de atividade física [9], que de acordo com a Organização Mundial da Saúde, crianças e adolescentes, com idade entre 5 e 17 anos devem envolver-se em atividades físicas de intensidade moderada a vigorosa pelo menos 60 minutos por dia, acumulando assim, 300 minutos por semana [10].

Atualmente, a Educação Física no Ensino Médio, contemplada na nova Lei de Diretrizes e Bases da Educação Nacional (LDB) em seu artigo 26, no parágrafo $3^{\circ}$, estabelece: "A Educação Física integrada à proposta pedagógica da escola, é componente curricular da educação, ajustando-se às faixas etárias e as condições da população escolar, sendo facultativa nos cursos noturnos" [11].

Diante do exposto, investigar a temática em questão poderá fornecer informações relevantes para a elaboração de programas de intervenção junto à escola e comunidade, além de nortear estudos futuros com o objetivo de aumentar o nível de atividade física e redução do sedentarismo entre os jovens. Portanto, o objetivo do presente estudo foi analisar a associação entre a participação em aulas de Educação Física e atitudes positivas para a prática de atividade física em adolescentes do estado de Sergipe, Brasil.

\section{MATERIAL E MÉTODOS}

Este estudo é uma análise secundária dos dados de um levantamento epidemiológico de delineamento transversal intitulado Condições de Vida e Condutas de Saúde em Adolescentes Residentes em Áreas Rurais e Urbanas no Estado de Sergipe, desenvolvido em 2011, pelo Grupo de Pesquisa em Educação Física e Saúde, do Instituto Federal de Educação, Ciência e Tecnologia de Sergipe (GPEFiS/IFS).

Este projeto foi aprovado pelo Comitê de Ética em Pesquisa com Seres Humanos do Hospital Universitário da Universidade Federal de Sergipe (CEP/UFS) sob o número do protocolo $\mathrm{N}^{\circ}$ CAAE - 2006.0.000.107-10.

De acordo com informações do Instituto Brasileiro de Geografia e Estatística [12], em 2010, o estado de Sergipe, localizado na região nordeste do Brasil, apresenta uma área da unidade territorial de 21.910,348 $\mathrm{km}^{2}$, com população total de 2.068 .031 pessoas, sendo 233.119 adolescentes (13-19 anos). O Estado é composto por 75 municípios, dividido geograficamente em oito territórios pela Secretaria de Estado do Planejamento.

A população alvo do estudo foi limitada por escolares de 14 a 19 anos de idade, de ambos os sexos, regularmente matriculados, nos períodos diurnos e noturnos, da rede estadual de ensino. Considerando os dados disponíveis na Secretaria de Estado da Educação, a matrícula do ensino médio, realizada em 2010, foi de 58.301 alunos em toda a Rede Estadual de Ensino, distribuídos em 155 unidades de ensino, que representavam aproximadamente $80 \%$ do total de estudantes do ensino médio em todo o estado.

Para compor a amostra da pesquisa foram sorteadas, inicialmente, as unidades de ensino, pela quantidade de alunos, distribuídas nos municípios de cada território. Em seguida, sortearam-se as turmas, por série e turno que seriam utilizadas para a coleta das informações. Para o cálculo deste planejamento amostral da pesquisa foi utilizado o processo de amostragem complexa disponível no software do SPSS (versão 15.0).

Em relação à estimativa do tamanho amostral na análise da prevalência para cada território, foi considerado o tamanho da população do território, a prevalência estimada em 50\% como a maior esperada, o intervalo de confiança em 95\%, o erro tolerável da amostragem em cinco pontos percentuais. Por se tratar de um processo amostral por conglomerado, multiplicou-se o tamanho amostral por 1,5 em função da correção do efeito do desenho (deff=1,5), estimando a necessidade 
de estudantes, para cada território (StatCalc. Epiinfo). Para não perder a representatividade amostral, foram acrescentados $20 \%$ de escolares devido a vários motivos, como: recusa do participante, idade maior ou menor do que a estabelecida nesse estudo, não ter respondido a questões importantes como sexo e idade, dentre outros.

Para a análise de associação foi considerado, além das informações descritas anteriormente, poder estatístico de $80 \%$ e Odds Ratio (OR) de 1,2. A opção por estes critérios no dimensionamento amostral decorreu da falta de conhecimento sobre a prevalência dos vários fatores que seriam investigados na população alvo, além da necessidade de atender aos diversos objetivos do projeto de pesquisa. O cálculo resultou em uma amostra mínima de 3875 adolescentes para todo o Estado.

$\mathrm{Na}$ seleção da amostra recorreu-se ao processo de amostragem por conglomerado em dois estágios: 1) realizou-se o processo de amostragem estratificada proporcional ao território (conglomerado) e porte da escola $(1=$ até 199 alunos; $2=200-499$ alunos; $3=500+$ alunos), segundo critério do Instituto Nacional de Estudos e Pesquisas Educacionais Anísio Teixeira (INEP) [13].

Desse modo, para que todos os territórios fossem contemplados, representativamente, com os três portes dos colégios, estabeleceu-se como critério o sorteio de $25 \%$ das unidades de ensino do Estado (155 unidades de ensino), totalizando 39 colégios distribuídos em 27 municípios; 2) selecionaram-se as turmas, de acordo com a série e o turno de estudo, mediante a utilização do processo aleatório simples, considerando uma média de 25 alunos por turma.

A participação dos adolescentes na pesquisa foi voluntária e anônima, adotando-se, além da utilização do termo de consentimento na forma negativa (Parental passive consent form) os seguintes critérios de inclusão: estar regularmente matriculado nas turmas de $1^{\circ}$ ao $3^{\circ}$ ano do ensino médio das escolas selecionadas; estar presente no momento da aplicação do instrumento; preencher adequadamente o questionário distribuído; ter idade entre 14 e 19 anos. Os diretores das escolas também assinaram um Termo de Consentimento Livre e Esclarecido autorizando a participação das instituições na pesquisa.

A coleta das informações foi realizada por professores de Educação Física, devidamente treinados quanto: ao domínio das questões do instrumento, forma de aplicação, supervisão e análise dos questionários após a aplicação. Na própria sala de aula ocorreu a aplicação do questionário, sendo dois professores por turma, para que pudessem esclarecer dúvidas e auxiliar no preenchimento das informações. A média de tempo da aplicação do questionário foi de 45 minutos. O instrumento utilizado foi uma versão de um questionário proposto pela Organização Mundial da Saúde (Global School - based Student Health Survey - GSHS/OMS) [14].

Neste estudo as variáveis dependentes foram: 1) realizar atividade física no tempo livre; 2) preferir atividades de lazer fisicamente ativas, por meio de duas questões objetivas extraídas do questionário: 1) "Você realiza, regularmente, algum tipo de atividade física no seu tempo livre, ou seja quando não está na escola ou no trabalho, como exercícios, esportes, danças ou artes marciais?"; 2) "Qual a atividade de lazer de sua preferência?". A variável "realizar atividade no tempo livre" apresentava duas opções de resposta (sim ou não) e "preferir atividades de lazer fisicamente ativas" apresentava nove opções de resposta, posteriormente classificadas em ativas (praticar esportes, fazer exercícios, nadar, pedalar) ou sedentárias (jogar dominó ou cartas, assistir TV, jogar videogame, usar o computador, conversar com os amigos).

A variável independente foi a "participação em aulas de Educação Física". Os adolescentes classificados como participantes foram aqueles que relataram participar em pelo menos uma aula de Educação Física por semana.

Foram usados o software Intelligent Character Recognition-Teleform (HS Informática, Rio de Janeiro, Brazil) e o scanner FI-6230 (Fujitsu, Tokyo, Japan) para realizar a tabulação dos dados por meio de leitora óptica. Após esta etapa os questionários que mostraram problemas como rasuras, foram conferidos e os erros corrigidos manualmente.

A análise dos dados foi realizada no programa SPSS para Windows (versão 15.0). Foi realizada a análise descritiva através do cálculo das prevalências e intervalos de $95 \%$ de confiança (IC95\%) das variáveis de interesse do estudo. Para a análise bivariada da relação do desfecho com as variáveis independentes utilizou-se o teste do qui-quadrado. $\mathrm{Na}$ análise multivariável foi usada a regressão logística binária, que representa como medida de associação a razão de chance 
(Odds Ratio). As variáveis com valores de $\mathrm{p}<0,20$ na análise multivariável bruta foram mantidas para serem ajustadas. Consideraram-se significativamente associadas ao desfecho, as variáveis cujo valor $p$ foi inferior a 0,05 .

\section{RESULTADOS}

Participaram da amostra final 3.992 escolares (38,7 \% masculino) de 14 a 19 anos. As características socioeconômicas e demográficas da amostra são apresentadas na tabela 1 . Verifica-se que a maioria dos jovens estavam matriculados no $1^{0}$ ano do Ensino Médio (41,3\%), nos turnos matutino e vespertino $(66,5 \%)$ e apresentaram atraso escolar em um ou mais anos em relação a idade (67,5\%). Além disso, encontrou-se baixa escolaridade da mãe $(62,0 \%$ não concluíram o Ensino Médio) e elevada prevalência da renda familiar em até dois salários mínimos $(71,0 \%)$.

Tabela 1: Características demográficas e socioeconômicas da amostra (n=3992). Sergipe, Brasil, 2011.

\begin{tabular}{|c|c|c|c|}
\hline Variáveis & Categorias & $\%(\mathrm{IC} 95 \%)$ & $\mathrm{n}$ \\
\hline \multicolumn{4}{|l|}{ Sexo } \\
\hline & Masculino & $38,7(37,2-40,2)$ & 1544 \\
\hline & Feminino & $61,3(59,8-62,8)$ & 2448 \\
\hline \multicolumn{4}{|l|}{ Faixa etária } \\
\hline & $14-15$ anos & $18,3(17,1-19,5)$ & 729 \\
\hline & 16-17 anos & $51,8(50,3-53,4)$ & 2069 \\
\hline & 18-19 anos & $29,9(28,5-31,3)$ & 1194 \\
\hline \multicolumn{4}{|c|}{ Série do Adolescente } \\
\hline & $1^{0}$ ano do $\mathrm{EM}^{1}$ & $41,3(39,8-42,8)$ & 1650 \\
\hline & $2^{0}$ ano do $\mathrm{EM}^{1}$ & $33,6(32,1-35,1)$ & 1343 \\
\hline & $3^{0}$ ano do $\mathrm{EM}^{1}$ & $25,0(23,7-26,3)$ & 999 \\
\hline \multicolumn{4}{|l|}{ Turno de estudo } \\
\hline & Diurno & $66,5(65,0-68,0)$ & 2653 \\
\hline & Noturno & $33,5(32,0-35,0)$ & 1339 \\
\hline \multicolumn{4}{|c|}{ Defasagem série-idade } \\
\hline & Aluno regular & $32,5(31,1-34,0)$ & 1297 \\
\hline & 1-2 anos de atraso & $49,2(47,7-50,8)$ & 1964 \\
\hline & $\geq 3$ anos de atraso & $10,0(9,1-11,0)$ & 398 \\
\hline & Adiantado & $8,3(7,4-9,2)$ & 333 \\
\hline \multicolumn{4}{|l|}{ Local de domicílio } \\
\hline & Área urbana & $61,8(60,3-63,3)$ & 2466 \\
\hline & Área rural & $38,2(36,7-39,7)$ & 1526 \\
\hline \multicolumn{4}{|c|}{ Escolaridade da mãe } \\
\hline & Não estudou & $11,4(10,4-12,4)$ & 456 \\
\hline & Não concluiu o $\mathrm{EF}^{2}$ & $50,6(49,1-52,2)$ & 2019 \\
\hline & Concluiu o $\mathrm{EF}^{2}$ & $10,1(9,2-11,0)$ & 403 \\
\hline & Concluiu o $\mathrm{EM}^{1}$ & $15,1(14,0-16,2)$ & 601 \\
\hline & Concluiu o $\mathrm{ES}^{3}$ & $7,4(6,6-8,2)$ & 295 \\
\hline & Não sabia & $5,5(4,8-6,2)$ & 218 \\
\hline \multicolumn{4}{|l|}{ Renda Familiar } \\
\hline & $<1 \mathrm{SM}^{4}$ & $32,1(30,7-33,6)$ & 1283 \\
\hline & 1 a $2 \mathrm{SM}^{4}$ & $38,9(37,4-40,4)$ & 1554 \\
\hline & $>2 \mathrm{SM}^{4}$ & $26,8(25,4-28,2)$ & 1068 \\
\hline & Não sabia & $2,2(1,7-2,7)$ & 87 \\
\hline
\end{tabular}

${ }^{1} \mathrm{EM}$, ensino médio; ${ }^{2} \mathrm{EF}$, ensino fundamental; ${ }^{3} \mathrm{ES}$, ensino superior; ${ }^{4} \mathrm{SM}$, salário mínimo.

As figuras 1 e 2 mostram maiores prevalências em atitudes positivas para a atividade física em jovens, de ambos os sexos, participantes de aulas de Educação Física. Verificou-se que 
os jovens que participam de aulas de Educação Física preferem atividades mais ativas e realizam mais atividades físicas no lazer, sendo maiores entre escolares do sexo masculino.

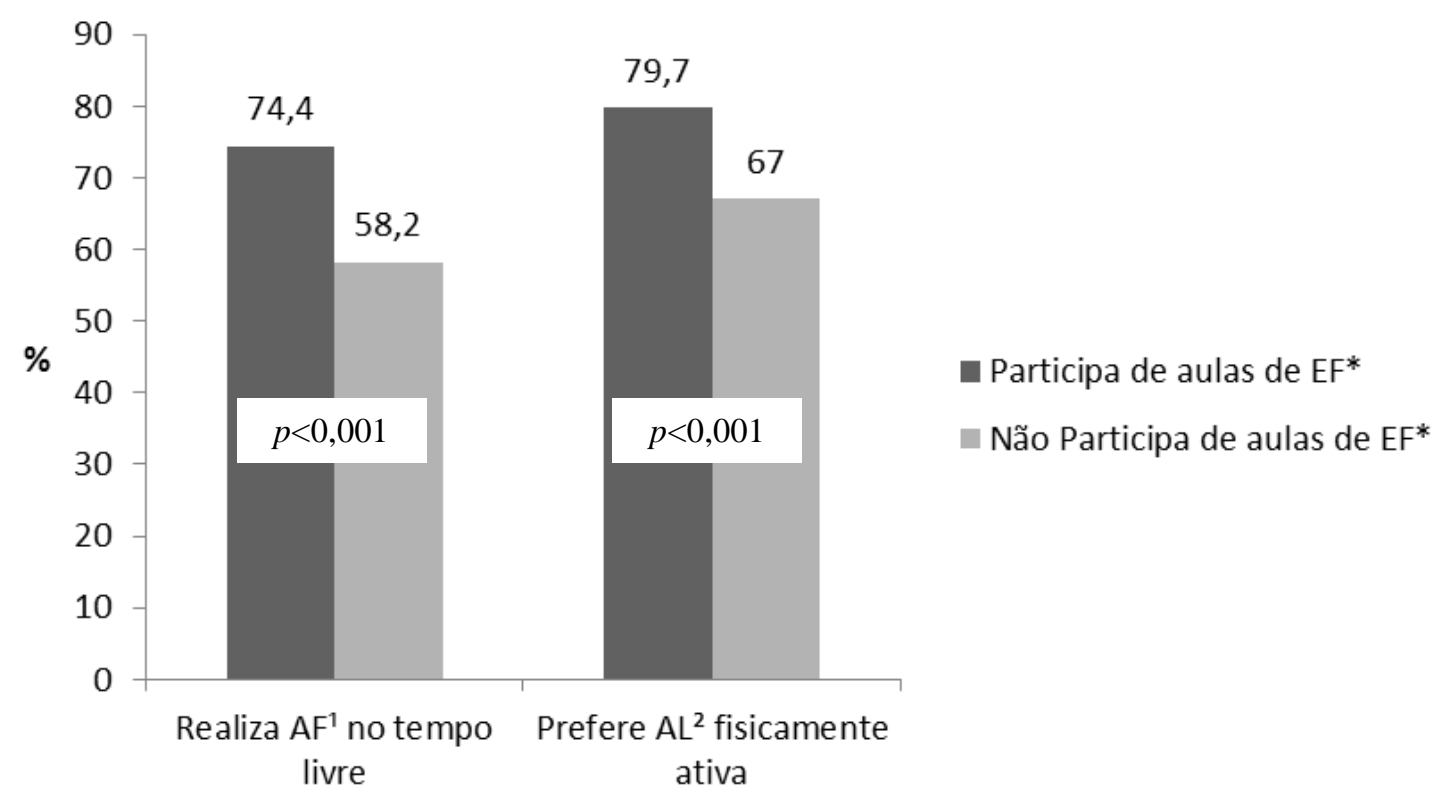

Figura 1: Prevalência de atitudes positivas para a prática de atividade física de acordo com a participação em aulas de educação física, em adolescentes do sexo masculino. Sergipe, Brasil, 2011. $A F^{1}$, atividade física; $A L^{2}$, atividade de lazer. $E F^{*}$, Educação Física

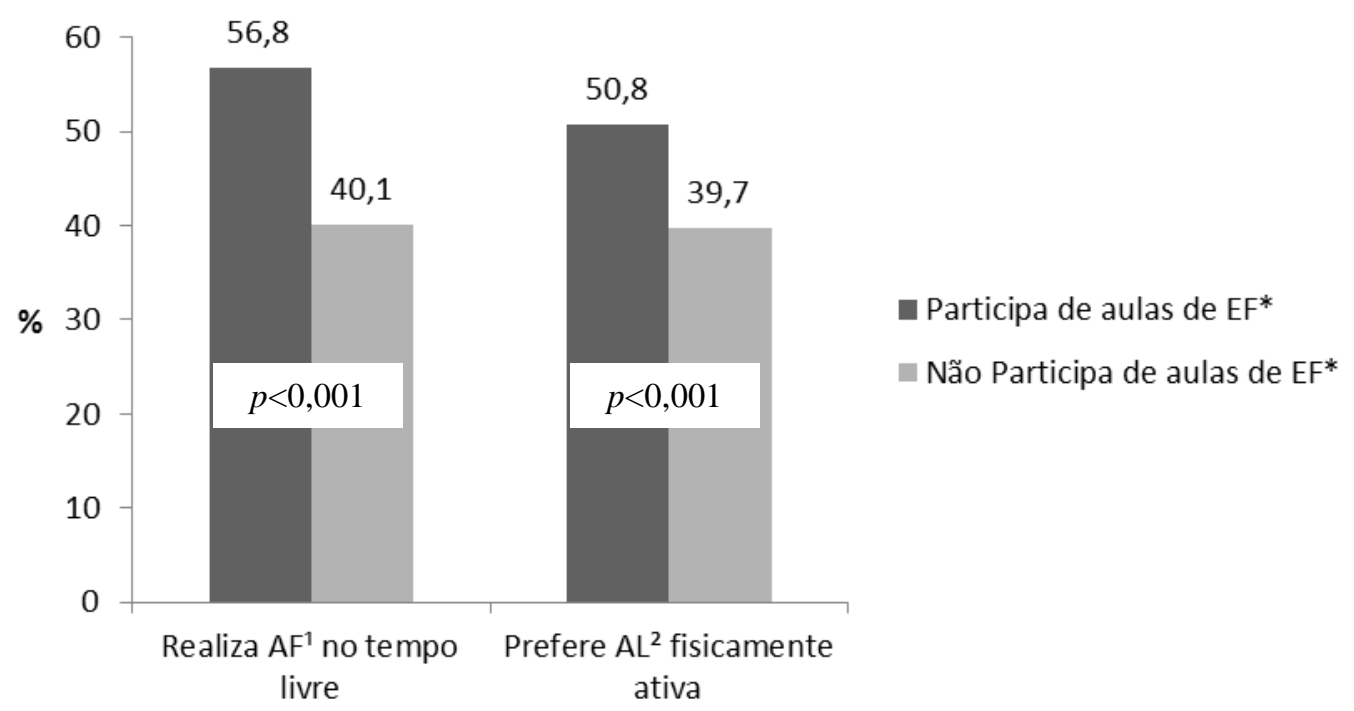

Figura 2: Prevalência de atitudes positivas para a prática de atividade física de acordo com a participação em aulas de educação física, entre adolescentes do sexo feminino. Sergipe, Brasil, 2011. $A F^{l}$, atividade física; $A L^{2}$, atividade de lazer. $E F^{*}$, Educação Física.

A tabela 2 mostra a análise bruta e ajustada da associação entre atitudes positivas e a participação nas aulas de Educação Física dos adolescentes. Verifica-se, após ajuste, que adolescentes participantes em aulas de Educação Física apresentaram maior chance de preferir atividades físicas a sedentárias no lazer $(\mathrm{OR}=1,69$; IC95\%: 1,45-1,96) e de serem ativos no seu 
tempo livre (OR=2,00; IC95\%: 1,72-2,31) em comparação aos seus pares que não participam das aulas.

Tabela 2: Análise bruta e ajustada da associação entre atitudes positivas para a prática de atividades fisicas e participação em aulas de Educação Física. Sergipe, Brasil, 2011.

\begin{tabular}{|c|c|c|c|}
\hline \multirow[b]{2}{*}{ Variável } & \multicolumn{3}{|c|}{ Atitudes positivas para a prática de atividade física } \\
\hline & Categoria & $\begin{array}{c}\text { OR Bruta } \\
\text { (IC95\%) }\end{array}$ & $\begin{array}{l}\text { OR Ajustada }{ }^{\mathbf{a}} \\
\text { (IC } 95 \%)\end{array}$ \\
\hline & \multicolumn{3}{|c|}{ Preferir Atividades Físicas a Sedentárias no Lazer } \\
\hline \multirow{3}{*}{${ }^{1} \mathbf{P A E F}$} & $\begin{array}{l}\text { Não } \\
\text { Sim }\end{array}$ & $\frac{1}{177(1.54-204)}$ & $\frac{1}{159(145-106)}$ \\
\hline & \multicolumn{3}{|c|}{ Realizar Atividades Físicas no Tempo Livre } \\
\hline & $\begin{array}{l}\text { Não } \\
\text { Sim }\end{array}$ & $\begin{array}{c}1 \\
2,08(1,80-2,41)\end{array}$ & $\begin{array}{c}1 \\
2,00(1,72-2,31)\end{array}$ \\
\hline
\end{tabular}

${ }^{1}$ PAEF, participação em aulas de Educação Física ( $\geq 1$ vez por semana); IC, Intervalo de Confiança; ${ }^{a}$ ajustada para sexo e idade.

\section{DISCUSSÃO}

Este estudo analisou as possíveis associações entre participação em aulas de Educação Física e atitudes positivas para a prática de atividade física em adolescentes do estado de Sergipe, região Nordeste do Brasil. Os resultados do estudo mostraram que 75\% (79,2\% masculino; $72,5 \%$ feminino) dos escolares participavam frequentemente de aulas de Educação Física. Essa prevalência é inferior a encontrada entre escolares Poloneses (96,0\%) [15], semelhante a do Sul do Brasil (78,4\% masculino; 65,7\% feminino) [16] e maior que nos Estados Unidos (28,4\%) [17], Canadá $(26,9 \%)$ [18]. Tais resultados configuram a necessidade de motivar os adolescentes a participarem de aulas de Educação Física, bem como, se realizar intervenções nas escolas com o intuito de adquirir benefícios à saúde.

Observou-se, também, que os jovens que frequentemente participaram de aulas de Educação Física apresentam melhores atitudes positivas para a prática de atividades físicas. Os benefícios da prática de atividades físicas são bem documentados na literatura, similarmente, sabe-se que a participação em aulas de Educação Física pode proporcionar substanciais benfeitorias à saúde física e mental em escolares [19]. Apesar de algumas investigações apontarem para o fato que aulas de Educação Física não previnem o declínio de atividades físicas da adolescência para a vida adulta [20], é sabido que jovens que participam de aulas de Educação Física aumentam significativamente o tempo total gasto em atividades físicas de moderadas a vigorosas durante a semana [21] e o $\mathrm{VO}_{2}$ máximo, além de reduzirem o tempo de exposição a comportamentos sedentários [22]. Da mesma forma, jovens que praticam esportes de forma organizada são mais prováveis para atingirem as recomendações internacionais à prática de atividades físicas, seja moderada, vigorosa e/ou total [23].

A prevalência de adolescentes participantes de aulas de Educação Física que responderam preferir atividades físicas no lazer foi de 79,7\%, no masculino e 50,8\%, no feminino, resultados semelhantes ao do estado de Pernambuco para o masculino $74,7 \%$, e diferente para o feminino, 28,2\% [24]. Investigações apontam que adolescentes tendem a preferir praticar mais de um tipo de atividade física, preferencialmente, ginástica e esportes coletivos, com amigos e fora de casa como forma de serem mais ativos [25]. Corroborando a descoberta desse estudo, este fato parece ocorrer com maior frequência quando os jovens adquirem habilidades motoras adequadas a sua prática, sendo diferente entre os sexos, aspecto que podem ser desenvolvidos com propriedade nas aulas de Educação Física Escolar.

Outra evidência apresentada nesse trabalho descreve que os escolares que participam de aulas de Educação Física relataram realizar, em seu tempo de lazer, atividades mais ativas em 
relação às sedentárias. A prevalência de adolescentes que responderam realizar atividades físicas no lazer foi de 79,7\% no masculino e 50,8\%, no feminino, resultados semelhantes ao do estado de Pernambuco, $87,6 \%$, no masculino e $80,0 \%$ no feminino [24]. Provável explicação para este achado é que a maior variabilidade na oferta de equipamentos e tipos de atividades físicas tende a elevar a adesão em relação a menor quantidade [26]. Desse modo, aulas de Educação Física Escolar desenvolvidas com qualidade apresentam forte potencial para aumentar a prática de atividades físicas no lazer em jovens, além disso, as unidades escolares podem impulsionar uma maior prática ofertando atividades esportivas extracurriculares, estimular o deslocamento ativo e orientar diferentes atividades em seus bairros [27].

Neste estudo, a associação entre participação em aulas de Educação Física e atitudes positivas para a prática de atividades físicas foi analisada por meio do componente quantitativo representado pela frequência as aulas. No entanto, deve-se observar a qualidade dos programas das aulas de Educação Física, especialmente, o tempo de engajamento em atividades físicas de intensidade moderada a vigorosa durante as aulas. Estudos revelam que os esforços físicos realizados nas aulas de Educação Física, em sua maioria, são caracterizados como sendo de curta duração e baixa intensidade [28,29] em relação às recomendações atuais [30], que preconizam pelo menos 50\% do tempo total das aulas em intensidade moderada a vigorosa. Sendo assim, os programas de Educação Física escolar, que não atendem a essas diretrizes podem inviabilizar o alcance dos níveis recomendados de atividade física para a saúde e insuficiente para influenciar a prática de atividade física tanto fora do ambiente escolar quanto na vida adulta.

Esta investigação apresentou as seguintes limitações que devem ser consideradas: a) a generalização dos dados para todos os adolescentes do estado pode ser visto com cautela devido o estudo ser restrito aos escolares da rede estadual de ensino; b) a possibilidade de causalidade reversa em virtude do tipo de delineamento transversal usado na pesquisa. Por outro lado, apresentou os seguintes pontos positivos: a) o estudo foi conduzido com um número relativamente grande de sujeitos, garantindo suficiente poder estatístico às análises; b) abrangência e cuidados no dimensionamento e seleção da amostra possibilitando a extrapolação dos resultados para a população alvo do estudo.

\section{CONCLUSÃO}

Em síntese, a participação em aulas de educação física foi um fator associado às atitudes positivas para a prática de atividade física entre os adolescentes investigados. Nesse sentido, a escola, por meio das aulas de Educação Física, pode oferecer oportunidades aos jovens de experimentarem e apreciarem um estilo de vida ativo. Sendo assim, são necessárias políticas públicas que garantam um número adequado de aulas de Educação Física por semana, com conteúdos voltados para o cotidiano dos escolares e práticas corporais diversificadas. Além disso, devem-se atender as recomendações do nível suficiente de atividade física durante as aulas para promover saúde e mudanças positivas para a prática de atividade física fora do ambiente escolar.

\section{AGRADECIMENTOS}

Este trabalho contou com o apoio da Capes (Coordenação de Aperfeiçoamento de Pessoal de Nível Superior), da Universidade Federal de Sergipe (UFS) e do Instituto Federal de Sergipe (IFS). Os autores agradecem aos alunos dos 39 colégios que participaram do projeto.

\section{REFERÊNCIAS BIBLIOGRÁFICAS}

1. Hallal PC, Victora CG, Azevedo MR, Wells JCK. Adolescent physical activity and health: a systematic review. Sports Med 2006;36(12):1019-30.

2. U.S. Department of Health and Human Services. Healthy people 2000: national health promotion and disease prevention objectives. In. Washington: US Department of Health and Human Services 1991. 
3. U.S. Department of Health and Human Services. Physical activity and health: A report of the Surgeon General. Atlanta, GA: U.S. Department of Health and Human Services, Center for Disease Control and Prevention, National Center for Chronic Disease Prevention and Health Promotion; 1996.

4. Parsons TJ, Power C, Logan S, Summerbell CD. Childhood predictors of Adolescent PA also seems to improve self-es- adult obesity: a systematic review. Int J Obes Relat Metab teem, and to increase lung function among cystic Disord 1999;23(8):S1-107.

5. Azevedo MR, Araújo CL, Silva MC, Hallal PC. Tracking of physical activity from adolescence to adulthood: a population-based study. Rev Saúde Publica 2007;41:69-75.

6. Martins MO, Cavalcante VLF, Holanda GS, Oliveira CG, Maia FES, Meneses Júnior JR et al. Associação entre comportamento sedentário e fatores psicossociais e ambientais em adolescentes da região nordeste do Brasil. Rev Bras Ativ Fis e Saúde 2012;17(2):143-50.

7. Chen S, Kim Y, Gao Z. The contributing role of physical education in youth's daily physical activity and sedentary behavior. BMC Public Health 2014;14:110 http://www.biomedcentral.com/1471-2458/14/110.

8. Lonsdale C, Rosenkranz RR, Sanders T, Peralta LR, Bennie A, Jackson B, Taylor IM, Lubans DR. A cluster randomized controlled trial of strategies to increase adolescents' physical activity and motivation in physical education: Results of the Motivating Active Learning in Physical Education (MALP) trial. Preventive Medicine 2013;57:696-702.

9. Fairclough S, Stratton G. Physical education makes you fit and healthy'. Physical education's contribution to young people's physical activity levels. Health Educ. Res 2005;20:14-23.

10.World Health Organization. Global recommendations on physical activity for health. Geneva: World Health Organization; 2010.

11. BRASIL. Presidência da República. Lei no 9.394, de 20 de dezembro de 1996. Diário Oficial, Brasília, 23 dez. 1996.

12. INSTITUTO BRASILEIRO DE GEOGRAFIA E ESTATÍSTICA. Pesquisa sobre dados demográficos do Estado de Sergipe 2008. Endereço eletrônico: http://www.ibge.gov.br.

13. Soares Neto JJ, Karino CA, Jesus GR, Andrade DF. A infraestrutura das escolas públicas brasileiras de pequeno porte. Rev do Serviço Público Brasília. 2013;64(3):377-91.

14. World Health Organization. Global School-Based Student Health Survey. Atlanta: World Health Organization [citado 1 abr. 2013]. Disponível em: http://www.cdc.gov/GSHS/.

15. Wojtyła-Buciora P, Stawińska-Witoszyńska B, Wojtyła K, Klimberg A, Wojtyła C, Wojtyła A, Samolczyk-Wanyura D, Marcinkowski JT. Assessing physical activity and sedentary lifestyle behaviours for children and adolescents living in a district of Poland. What are the key determinants for improving health? Annals of Agricultural and Environmental Medicine 21, (3) 606-612, 2014.

16. Coledam DHC, Ferraiol PF, Pires Junior R, dos-Santos JW, Oliveira AR. Prática esportiva e participação nas aulas de educação física: fatores associados em estudantes de Londrina, Paraná, Brasil. Cad. Saúde Pública. 2014;30(3):533-45.

17. Centers for Disease Control and Prevention (CDC). Participation in high school physical education-United States, 1991-2003. MMWR Morb Mortal Wkly Rep. 2004 Sep 17;53(36):844-7.

18. Centers for Disease Control and Prevention (CDC). Participation in high school physical education-Ontario, Canada, 1999-2005. MMWR Morb Mortal Wkly Rep. 2007 Jan 26;56(3):52-4.

19. Hills AP, Dengel DR, Lubans DR. Supporting Public Health Priorities: Recommendations for Physical Education and Physical Activity Promotion in Schools. Progress in Cardiovascular Diseases. 5 (7) 2015, 368-374. http://dx.doi.org/10.1016/j.pcad.2014.09.010.

20. Palakshappa D, Virudachalam S, Oreskovic NM, Goodman E. Adolescent Physical Education Class Participation as a Predictor for Adult Physical Activity. Child Obes. 2015 Oct;11(5):616-23. doi: 10.1089/chi.2015.0024. Epub 2015 Sep 8.

21. Lonsdale C, Rosenkranz RR, Peralta LR, Bennie A, Fahey P, Lubans DR. A systematic review and meta-analysis of interventions designed to increase moderate-to-vigorous physical activity in school physical education lessons. Preventive Medicine 2013; 56(2): 152-161 doi: 10.1016/j.ypmed.2012.12.00.

22. Dobbins M, Husson H, DeCorby K, LaRocca RL. School-based physical activity programs for promoting physical activity and fitness in children and adolescents aged 6 to 18. Cochrane Database Syst Rev. 2013 Feb 28;2:CD007651. doi: 10.1002/14651858.CD007651.pub2.

23. Matques A, Ekelund U, Sardinha LB. Associations between organized sports participation and objectively measured physical activity, sedentary time and weight status in youth. J Sci Med Sport. 2016 Feb;19(2):154-7. doi: 10.1016/j.jsams.2015.02.007.

24. Hardman CM, Barros SSH, Andrade MLSS, Nascimento JV, Nahas MK, Barros MVG. Participação nas aulas de educação física e indicadores de atitudes relacionadas à atividade física em adolescentes. Rev Bras Educ Fís Esporte, (São Paulo) 2013 Out-Dez; 27(4):623-31

25. Corder K1, Atkin AJ, Ekelund U, van Sluijs EM. What do adolescents want in order to become more active? BMC Public Health. 2013 Aug 5;13:718. doi: 10.1186/1471-2458-13-718. 
26. Juvancic-Heltzel JA, Glickman EL, Barkley JE. The effect of variety on physical activity: a crosssectional study. J Strength Cond Res. 2013 Jan;27(1):244-51. doi: 10.1519/JSC.0b013e3182518010.

27. Trudeau F, Shephard RJ. Contribution of school programmes to physical activity levels and attitudes in children and adults. Sports Med. 2005;35(2):89-105.

28. Scruggs PW, Mungen JD, Yonsin O. Quantifying moderate to vigorous physical activity in high scholl physical education: a pedometer steps/minute standard. Measurement in Physical Education \& exercise Science 2010;14(2):104-15.

29. Gordon-Larsen P, McMurray RG, Popkin BM. Determinants of adolescent physical activity and inactivity patterns.Pediatrics 200;105(6):83-91.

30. Khan LK, Sobush K, Keener D, Goodman K, Lowry A, Kakietek J et al. Center for Disease Control and Prevention. MMWR Recomm Rep 2009;24(58):1-26. 